EESTI NSV TEADUSTE AKADEEMIA TOIMETISED. 28. KOIDE

FOOSIKA * MATEMAATIKA. 1979, NR. 2

ИЗВЕСТИЯ АКАДЕМИИ НАУК ЭСТОНСКОИ ССР. ТОМ 28 ФИЗИКА * МАТЕМАТИКА. 1979, № 2

\title{
К ТЕОРИИ ПРИЗЕМНОГО СЛОЯ АТМОСФЕРЫ
}

\author{
(Представлена Н. Эпиком)
}

В настоящее время расчет характеристик приземного слоя атмосферы базируется обычно на теории подобия Монина-Обухова $\left[{ }^{1,2}\right]$, допускающей наиболее общий подход к описанию/ турбулентности в термически стратифицированной среде. Согласно этой теории, статистический режим турбулентности в приземном слое атмосферы определяется лишь гремя размерными характеристиками: напряжением трения на поверхности (турбулентным потоком импульса) $\tau_{w}$, турбулентным потоком гепла $H_{w}$ и параметром плавучести $\beta=g / T(g-$ ускорение силы тяжести, $T$ - абсолютная температура на данной высоте). Из этих параметров составляется характерный масштаб

$$
L=-\frac{\tau_{w} / \varrho}{\chi \beta H_{w} / c_{p} \varrho}=U_{*}^{2} / \chi^{2} \beta T
$$

называемый масштабом длины Монина-Обухова. Здесь е - плотность, $c_{p}$ - удельная теплоемкость при постоянном давлении, $\chi-$ константа Кармана, $U_{*}=\left(\tau_{w} / \mathrm{Q}\right)^{1 / 2}-$ динамическая скорость, $T_{*}=$ $=-H_{w} / \chi c_{p} U_{*}$ - динамическая температура.

Из теории подобия следует, в частности, что вертикальные градиенты осредненных скорости ветра $U$ и температуры $T$ могут быть выражены следующим образом:

$$
\partial U / \partial z=(U * / \chi z) \varphi_{\mathrm{M}}(\zeta), \quad \partial T / \partial z=(T * / z) \varphi_{\mathrm{T}}(\zeta),
$$

где $z$ - высота над земной поверхностью, $\zeta=z / L-$ параметр стратификации. Конкретный вид универсальных функций $\varphi_{\mathrm{M}}$ и $\varphi_{\mathrm{T}}$ определяется на основе эмпирического обобщения опытных данных либо с помощью тех или иных полуэмпирических моделей турбулентности.

В настоящей работе сделана попытка расчета характеристик приземного слоя атмосферы над ровной подстилающей поверхностью постоянной шероховатости на основе модели, развитой в работе $\left[{ }^{3}\right]$.

1. Рассмотрим прежде всего, как можно учесть влияние архимедовых сил на характеристики турбулентности пограничного слоя в рамках феноменологической модели $\left[{ }^{3}\right]$. В этой работе для плоско-параллельного течения несжимаемой жидкости было выведено балансовое уравнение для кинетической энергии первичной составляющей пульсации скорости в следующем виде:

$$
\left.U \frac{\partial q_{0}^{2} / 2}{\partial x}+W \frac{\partial q_{0} / 2}{\partial z}=-\frac{\partial}{\partial x} \overline{\left(u_{0} p / \varrho\right)}-\frac{\partial}{\partial z} \overline{\left(w_{0} p\right.} / \varrho\right)+\overline{u_{0} f_{x}}+\overline{w_{0} f_{z}}
$$


Здесь $U, W$ - компоненты вектора осредненной скорости $\vec{Q}$ по осям $x, z$ (ось $x$ соответствует основному направлению течения, ось $z$ перпендикулярна к $x$ в плоскости осредненного течения); $u_{0}$, $w_{0}-$ компоненты вектора первичной составляющей пульсации скорости $\vec{q}_{0}$; $p$ - пульсация давления; $f_{x}, f_{z}-$ компоненты вектора пульсационной составляющей архимедовой силы $\vec{f}$. С учетом аппроксимаций теории свободной конвекции имеем

$$
\vec{f}=\vec{g} t / T,
$$

где $t-$ пульсация избыточной температуры, $\vec{g}\left\{g_{x}, g_{z}\right\}-$ вектор ускорения силы тяжести.

Предположим, что

$$
t=t_{0}-l_{z}^{\prime}(\partial T / \partial z) .
$$

Здесь $t_{0}-$ первичная составляющая пульсации температуры, обусловленная пульсациями теплового потока от действующего на течение источника нагрева; $l_{z}^{\prime}=\alpha_{0} l_{z}, l_{z}-$ поперечная составляющая вектора линейного масштаба турбулентности $\vec{l} ; \alpha_{0}-$ коэффициент пропорциональности. Учитывая, что $t_{0}$ может коррелировать с $u_{0}$ и $w_{0}$ в основном через посредство архимедовых сил, можем записать

$$
\overline{u_{0} t_{0}}=k q_{0}\left|t_{0}\right| g_{x} / g, \quad \overline{w_{0} t_{0}}=k q_{0}\left|t_{0}\right| g_{z} / g,
$$

где $0 \leqslant k \leqslant 1-$ коэффициент корреляции. Этот коэффициент, вообще говоря, может зависеть от ряда факторов, в том числе и от термической стратификации течения. Очевидно, в случае безразличной стратификации $k=0$.

С учетом $(1.2)-(1.4)$ и в соответствии с изложенными в $\left[{ }^{3}\right]$ правилами осреднения получим в результате

$$
\overline{u_{0} f_{x}}=k q_{0}\left|t_{0}\right| g_{x}^{2} / g T, \quad \overline{w_{0} f_{z}}=k q_{0}\left|t_{0}\right| g_{z}^{2} / g T-\alpha_{0} N q_{0} l g_{z}(\partial T / \partial z) / T,
$$

где $N$ - коэффициент, связанный с распределением плотности вероятности пульсации скорости в различных направлениях. Из (1.5) ясно видно, что если архимедова сила действует поперек течения, она оказывает на его турбулентную структуру значительно большее влияние, нежели будучи направленной вдоль него. Этот вывод согласуется с уже известными данными $\left[{ }^{4}\right]$ :

На основании сказанного выше и учитывая выведенные в $\left[{ }^{3}\right]$ выражения для пульсаций скорости, можем получить также формулу для турбулентного потока тепла, входящую в уравнение баланса термической энергии:

$$
t w=k q_{0}\left|t_{0}\right| g_{z} / g-\alpha_{0} N q_{0} l(\partial T / \partial z) .
$$

2. Рассмотрим теперь непосредственно течение в приземном слое атмосферы, в пределах которого турбулентные потоки импульса и тепла обычно принято считать постоянными по высоте, т. е.

$$
\begin{aligned}
& K_{\mathrm{M}}(d U / d z)=\tau_{w} / \mathrm{Q}=U^{2}=\text { const, } \\
& K_{\mathrm{T}}(d T / d z)=-H_{w} / c_{p \varrho}=\chi U \cdot T \cdot=\text { const. }
\end{aligned}
$$

Здесь $K_{\mathrm{M}}, K_{\mathrm{T}}-$ коэффициенты турбулентного переноса импульса (турбулентная вязкость) н тепла. 
С учетом формул (1.5), принятого в [ $\left.{ }^{3}\right]$ допущения о диффузионном характере переноса турбулентной энергии пульсациями давления, а также предполагая отсутствие изменения любых характеристик течения в продольном направлении (следствием чего являются зависимости (2.1)), можем записать уравнение баланса кинетической энергии первичной составляющей (1.1) в данном случае в виде

$$
d\left[K_{e}\left(d q_{0}^{2} / d z\right)\right] / d z=-\beta\left[\alpha_{0} N q_{0} l(d T / d z)-k q_{0}\left|t_{0}\right|\right],
$$

где $K_{e}-$ коэффициент турбулентного переноса энергии. Согласно $\left[{ }^{3}\right]$, а также в соответствии с (1.6) получим

$$
\begin{aligned}
& K_{\mathrm{M}}=N q_{0} l, \quad K_{e}=N q_{0} l / A, \\
& K_{\mathrm{T}}=\alpha_{0} N q_{0} l-k q_{0}\left|t_{0}\right| /(d T / d z),
\end{aligned}
$$

где $A-$ константа.

Интегрирование $(2.2)$ с учетом $(2.1),(2.3)$ и допущения

$$
N=n(z / l)
$$

(n- константа) дает

$$
q_{0}=q_{0 m}(1-M \zeta)^{1 / 3},
$$

где $q_{0 m}-$ условное значение $q_{0}$ при $z=0$ и

$$
M=\left(3 A / 2 \chi^{2}\right)\left(U_{*} / q_{0 m}\right)^{2}, \quad \chi=n\left(q_{0 m} / U_{*}\right) .
$$

Подставляя, наконец, (2.6) в (2.1) и учитывая (2.3), находим

$$
d U / d z=U * / \chi z(1-M \zeta)^{1 / 3}, \quad d T \cdot d d z=T_{*} / \alpha z(1-M \zeta)^{1 / 3},
$$

где $\alpha=K_{\mathrm{T}} / K_{\mathrm{M}}$.

Из сравнения формул (2.7) и (0.1) определяются аналитические выражения для функций $\varphi_{\mathrm{M}}$ и $\varphi_{\mathrm{T}}$ :

$$
\varphi_{\mathrm{M}}=1 /(1-M \zeta)^{1 / 3}, \quad \varphi_{\mathrm{T}}=1 / \alpha(1-M \zeta)^{1 / 3} .
$$

При $|M \zeta|<1$ функцию $\varphi_{M}$ можно разложить в ряд Тейлора. Сохраняя первые два члена разложения и интегрируя выражение для $d U / d z$ в (2.7), получим для малых $|\zeta|$ известный логарифмическо-линейный профиль скорости

$$
U-U_{1}\left(\zeta_{1}\right)=\left(U_{*} / \chi\right)\left[\ln \left(z / z_{1}\right)+x\left(\zeta-\zeta_{1}\right)\right],
$$

где $x=M / \zeta, U_{1}-$ скорость ветра на фиксированной высоте $z_{1}$ или $\zeta_{1}$.

В предельном случае сильной неустойчивости $M \zeta \ll-1$ непосредственным интегрированием (2.7) получим также известный «закон $-1 / 3 »$ :

$$
U-U_{1}=-\left(3 U \cdot L^{1 / 3} / M \zeta\right)\left(z^{-1 / s}-z_{1}^{-1 / s}\right) .
$$

В общем же случае интегрирование (2.7) приводит к следующему выражению для профиля скорости ветра:

$$
\begin{gathered}
U-U_{1}\left(\zeta_{1}\right)=\frac{U \cdot}{\chi}\left[\ln \left|\frac{1-Z}{1-Z_{1}}\right|\left(\frac{1+Z_{1}+Z_{1}^{2}}{1+Z+Z^{2}}\right)^{1 / 2}+\right. \\
\left.+\frac{1}{\sqrt{3}}\left(\operatorname{arctg} \frac{2 Z+1}{\sqrt{3}}-\operatorname{arctg} \frac{2 Z_{1}+1}{\sqrt{3}}\right)\right],
\end{gathered}
$$

где $Z=\varphi_{\mathrm{M}}, \quad Z_{1}=\left.Z\right|_{\zeta=\zeta_{1}}$, 
Очевидно, что формулы $(2.8),(2.10)$ и (2.11) справедливы лишь при $M \zeta<1$, т. е. при $\zeta<\zeta_{k}=1 / M$. Подставляя $(2.4)-(2.6)$ и $(2.8)$ в $(2.3)$, находим

$$
\begin{aligned}
& K_{\mathrm{M}}=\chi U * z / \varphi_{\mathrm{M}}, \\
& K_{\mathrm{T}}=\chi U \cdot\left[\alpha_{0} z-k\left|t_{0}\right| / n(d T / d z)\right] / \alpha \varphi_{\mathrm{T}},
\end{aligned}
$$

т. е. при $\zeta \rightarrow \zeta_{k}$ имеем $K_{\mathrm{M}} \rightarrow 0$ и $K_{\mathrm{T}} \rightarrow 0$. Таким образом, в рамках данной модели $\zeta_{k}$ представляет собой критическое значение параметра стратификации, при котором крупномасштабная турбулентная активность исчезает. В действительности же, согласно современным воззрениям, при достаточно высокой степени устойчивости приземного слоя турбулентное смешение определяется прежде всего мелкомасштабными пульсациями, а затем случайными внутренними гравитационными волнами $\left.{ }^{2}\right]$. Отсюда следует, что коэффициенты обмена $K_{\mathrm{M}}$ и $K_{\mathrm{T}}$ при $\zeta \rightarrow \zeta_{k}$ должны стремиться к некоторым конечным значениям $K^{\prime}$ м и $K^{\prime}$ т. Указанное обстоятельство может быть приближенно учтено формулами

$$
\begin{gathered}
K_{\mathrm{M}}=\left\{\begin{array}{lll}
N q_{0} l+K^{\prime}{ }_{\mathrm{M}} & \text { при } & \zeta<\zeta_{k}, \\
K^{\prime} \mathrm{M} & \text { при } & \zeta \geqslant \zeta_{k} ;
\end{array}\right. \\
K_{\mathrm{T}}=\left\{\begin{array}{llr}
\alpha_{0} N q_{0} l-k q_{0}\left|t_{0}\right| /(d T / d z)+K^{\prime}{ }_{\mathrm{T}} & \text { при } & \zeta<\zeta_{k}, \\
K_{\mathrm{T}}^{\prime} & \text { при } & \zeta \geqslant \zeta_{k} .
\end{array}\right.
\end{gathered}
$$

Тогда

$\varphi_{\mathrm{M}}= \begin{cases}1 /\left[(1-M \zeta)^{1 / 4}+K_{\mathrm{M}}^{\prime} / \chi U \cdot L \zeta\right] & \text { при } \zeta<\zeta_{k}, \\ \chi U \cdot L \zeta / K_{\mathrm{M}}^{\prime} & \text { при } \zeta \geqslant \zeta_{k}\end{cases}$

$\varphi_{\mathrm{T}}=\left\{\begin{array}{lr}1 /\left[\alpha_{0}(1-M \zeta)^{1 / 3}-k L(1-M \zeta)^{1 / 3}\left|t_{0}\right| / \zeta(d T / d z)+K_{\mathrm{T}}^{\prime} / \chi U \cdot L \zeta\right] & \text { при } \zeta<\zeta_{k}, \\ \chi U \cdot L \zeta / K_{\mathrm{T}}^{\prime} & \text { при } \zeta \geqslant \zeta_{k} .\end{array}\right.$

В соответствии с теорией подобия, при $\zeta=0$ должно быть $\varphi_{\mathrm{M}}(0)=$ $=\varphi_{\mathrm{T}}(0) / \alpha=1$. Функции (2.14) отвечают этому условию в том случае, если при $\zeta<\zeta_{k}$ величины $K_{\text {м }}^{\prime}$ и $K_{\text {т }}^{\prime}$ быстро уменьшаются, стремясь к нулю при $\zeta \rightarrow 0$. Считая, далее, что $K^{\prime}$ м при $\zeta>\zeta_{k}$ не зависит от высоты, получим с помощью (2.9) линейный закон распределения скорости ветра по высоте:

$$
U=\left(U_{*}^{2} / K_{\mathrm{M}}^{\prime}\right) z+\text { const. }
$$

Определим ориентировочно численное значение константы $M$ согласно (2.6). Входящее в эту зависимость отношение $q_{0 m} / U$. должно, в принципе, вычисляться на основе опытных данных по измерению турбулентности в пограничном слое у плоской стенки. Однако определение $q_{0 m}$ в непосредственной близости от нее сопряжено со значительными трудностями. Между тем сравнение таких опытных данных с данными по турбулентным характеристикам течений в каналах и трубах показывает, что вблизи стенки распределения среднеквадратичных значений пульсационных составляющих скорости $\overline{u^{2}}, \overline{v^{2}}, \overline{w^{2}}$ в количественном и качественном отношениях весьма близки для рассматриваемых типов течений. Это позволяет воспользоваться соответствующими данными для равномерного течения в каналах и трубах, где $q_{0}=q_{0 m}=$ const 


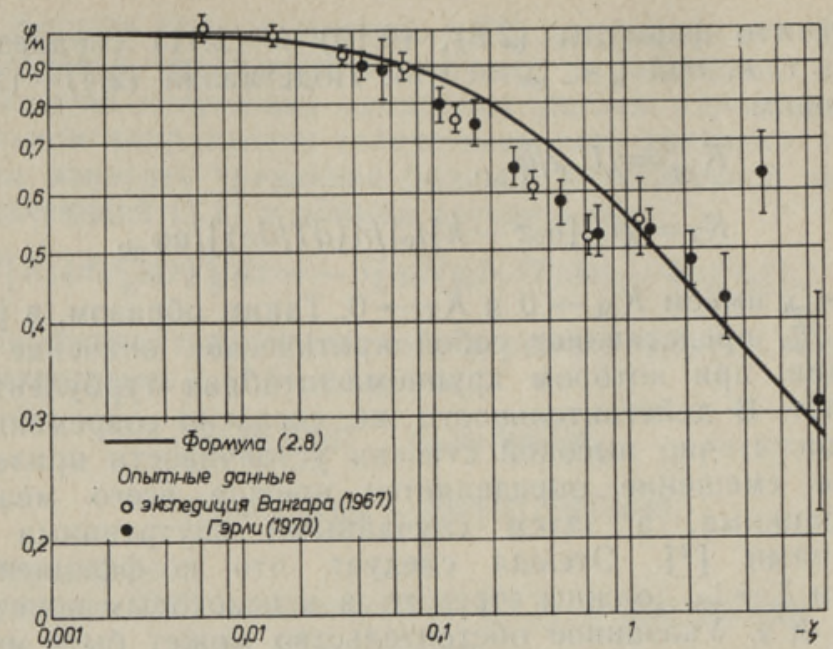

Pнс. 1.

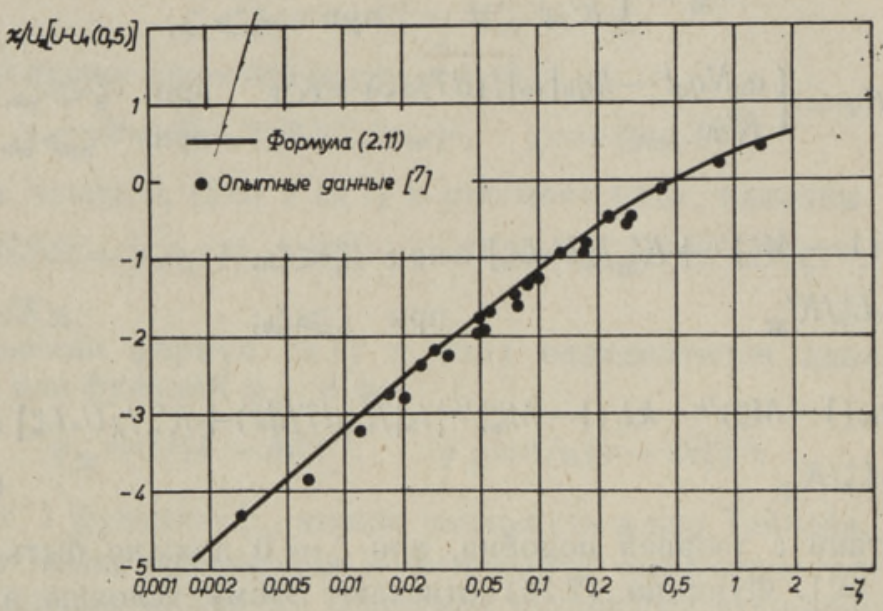

Рис. 2.

по всей ширине течения и $q_{0 \mathrm{~m}} / U \approx \approx 1,4\left[{ }^{5}\right]$. Принимая также $\chi=0,4$ и $A=1$, получим $M=4,7$. Отсюда $ж \approx 1,6$ и $\zeta_{k} \approx 0,21$. Разумеется, эти значения констант подлежат дальнейшему уточнению.

На рис. 1 показано сравнение расчетных значений $\varphi_{\mathbf{M}}$ с данными различных натурных измерений при неустойчивой стратификации $(\zeta<0)$, приведенными в [ [ $\left.{ }^{6}\right]$. Как видно, их удовлетворительное соответствие достигается при $\zeta>-0,1$ и $\zeta<-1$, в то время как при $-0,1>\zeta>-1$ расчетные значения фм оказываются несколько завышенными.

На рис. 2,3 рассчитанный по формуле (2.11) профиль скорости ветра сравнивается с профилями натурных измерений $[7,8]$ при неустойчивой и устойчивой стратификациях окружающей среды соответственно. Следует подчеркнуть, что удовлетворительное согласие между расчетными результатами и экспериментальными данными получено по единой теоретической формуле и при одинаковом значении эмпирической константы $M$ как для отрицательных, так и положительных значений, вплоть до $\zeta_{k}$. 


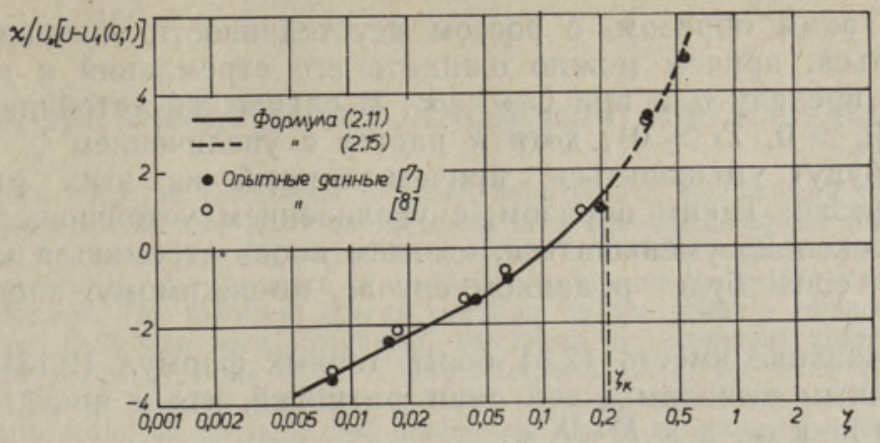

Рис. 3.

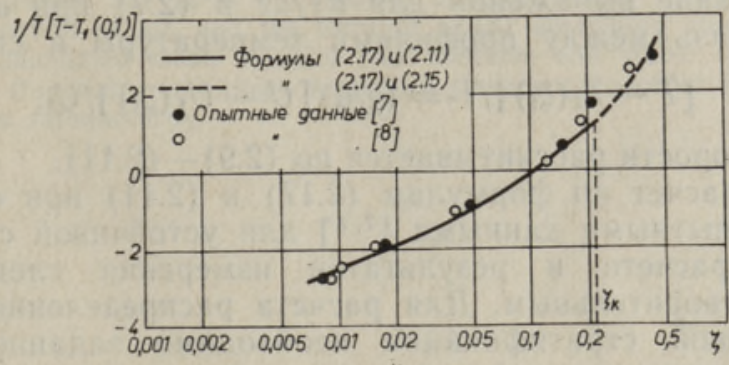

Рис. 4.

Формулы, аналогичные $(2.9)-(2.11)$, можно было бы получить и для вертикальных профилей температуры приземного слоя, если бы коэффициент а был константой. Однако, как явствует из большинства имеющихся опытных данных, $\alpha$ является в действительности функцией параметра $\zeta$, особенно при $\zeta<0[9,10]$.

С помощью формул $(2.3)-(2.7)$ может быть выведена следующая зависимость $\alpha$ от параметра стратификации $\zeta:$

$$
\alpha=\alpha_{0} /\left[1+k\left|t_{0}\right|(1-M \zeta)^{1 / 3} / n T \cdot\right] .
$$

В этом выражении $n-$ константа. Коэффициент $k$, как указывалось выше, должен зависеть от стратификации приземного слоя. Поскольку в рамках данной модели единственным безразмерным параметром, характеризующим стратификацию, является $\zeta$, то $k$ есть возрастающая функция $|\zeta|$. По определению, $t_{0}$ связана с пульсациями теплового потока от действующего на течение источника нагрева. Поскольку нагрев и охлаждение приземного слоя определяются, в основном, теплообменом с земной поверхности, $t_{0}$ должна быть обусловлена пульсациями теплового потока у поверхности. При этом непосредственно прилегающий к поверхности слой будет испытывать максимальное влияние пульсации теплового потока, а с высотой это влияние должно ослабевать. Иными словами, отношение $\left|t_{0}\right| / T$. должно быть, видимо, убывающей функцией $|\zeta|$.

В случае безразличной стратификации $(\zeta=0) \quad k=0$ и $\alpha=\alpha_{0}$. При неустойчивой стратификации $(\zeta<0, T .<0) k$ с ростом $|\zeta|$ увеличивается, стремясь, однако, к некоторой конечной величине, не превышающей единицы; беспредельный же рост $(1-M \zeta)^{1 / 3}$ компенсируется уменьшением отношения $\left|t_{0}\right| /|T \cdot|$, которое стремится к нулю при 
$|\zeta| \rightarrow \infty$. Таким образом, с ростом неустойчивости значение $\alpha$ будет увеличнваться, причем можно ожидать его стремления к некоторому конечному пределу $\alpha_{-\infty}$ при $\zeta \rightarrow-\infty$. В случае же устойчивой стратификации $(\zeta>0, T *>0)$, хотя $k$ растет с увеличением $\zeta,(1-M \zeta)^{1 / 3}$ и $\left|t_{0}\right| / T$ * будут уменьшаться, причем первый из этих множителей довольно резко. Таким образом, с увеличением устойчивости $\alpha$ будет сначала несколько уменьшаться, а затем вновь стремиться к $\alpha_{0}$. Допущение $\alpha=$ const будет в данном случае, по-видимому, вполне оправданным.

Использование вместо (2.3) более точных формул (2.13) приводит к аналогичным выводам с той лишь разницей, что $\alpha$ при $\zeta \rightarrow \zeta_{k}$ будет стремиться не к $\alpha_{0}$, а к $K_{\text {T }}^{\prime} / K^{\prime}$ м.

Сделанные выше выводы качественного характера, хотя и не вполне строгие, правильно отражают реальные изменения $\boldsymbol{\alpha}$. Это подтверждает большинство имеющихся результатов измерений $[2,4,9,10]$.

Интегрирование выражения для $d T / d z$ в (2.7) при $\alpha=$ const дает следующую связь между профилями температуры и скорости ветра:

$$
\left[T-T_{1}\left(\zeta_{1}\right)\right] / T *=(\chi / \alpha)\left[U-U_{1}\left(\zeta_{1}\right)\right] / U_{*},
$$

где профиль скорости рассчитывается по (2.9)-(2.11).

На рис. 4 расчет по формулам (2.17) и (2.11) при $\alpha=1,15$ сопоставляется с опытными данными $\left[{ }^{7,8}\right]$ для устойчивой стратификации. Согласование расчета и результатов измерения следует признать вполне удовлетворительным. Для расчета распределения температуры при неустойчивой стратификации необходимо задание зависимости $\alpha(\zeta)$, о количественном характере которой мнения различных исследователей существенно расходятся $[2,9,10]$.

\section{ЛИ ТЕ Р А Т У Р А}

1. Мон ин А. С., О б у хов А. И., ДАН СССР, 93, № 2, 223-226 (1953).

2. Мон ин А. С., Я глом А. М., Статистическая гидромеханика, ч. І., М., «Наука», 1965.

3. Э п ш т е й н А., Изв. АН ЭССР, Физ. Матем., 27, № 4, 413-422 (1978).

4. P a n of s k y, H. A., Ann. Rev. Fluid Mech., 6, 147-177 (1974).

5. Э п ш те й н А., Изв. АН ЭССР, Физ. Матем., 28, № 2, 132-137 (1979).

6. Pasquill, F., Atmospheric diffusion, New York-London-Sydney-Toronto, Halsted Press, 1974.

7. Г у р в и ч А. С., Изв. АН СССР, Физ. атмосф. и океана, 1, № 1; 55-65 (1965).

8. Bu s inger, J. A., W y n g a a rd, J. S., Yzumi, J., B r a d ley, E. F., J. Atm. Sci., 28, № 2, 181-189 (1971).

9. З и литинке вич С. С., Динамика пограничного слоя атмосферы, Л., Гидрометеоиздат, 1970.

10. Я гл о м А. М., Изв. АН СССР, Физ. атмосф. и океана, 10, № 6, 566-586 (1974).

Институт термофизики и электрофизики Академии наук Эстонской ССР
Поступила в редакцию $15 /$ XII 1978

\section{A. EPSTEIN}

\section{ATMOSFÄARI MAALÄHEDASE KIHI TEOORIAST}

Artiklis on käsitletud stratifitseeritud maalähedase õhukihi keskmise tuulekiiruse ja temperatuuri vertikaalsete profiilide arvutamist autori loodud fenomenaalse turbulentsusmudeli alusel. Arvutuslikul teel saadud tulemused kattuvad vaatlusandmetega rahuldavalt. 


\section{A. EPSTEIN}

\section{ON THE THEORY OF THE ATMOSPHERIC SURFACE LAYER}

The paper deals with the calculation of mean velocity and temperature profiles in the stratified atmospheric surface layer on the basis of a phenomenological model of turbulence proposed by the author $\left[{ }^{3}\right]$. In addition to the equations proposed in $\left[{ }^{3}\right]$, relations (1.5), (1.6) describing the influence of buoyancy forces on the properties of flow turbulence are derived. The turbulent energy equation for the primary component of velocity fluctuation $(2.2)$ has been integrated, assuming the «constant shear stress» and «constant thermal energy flux» (2.1). A single formula (2.11) for mean velocity profile as a function of the stability parameter $z / L(z-$ height above surface, $L-M o n i n-$ Obukhov length scale) at unstable and moderately stable conditions is deduced. In the case of a strong instability this formula reduces to the well-known «-1/3 law» $(2.10)$ and at near neutral stratification - to the «logarithmic+linear law» (2.9). Numerical values of the empirical constants as well as the critical value of $(z / L)$, at which the large scale turbulence vanishes, are found from the analysis of experimental data for channel and pipe flows. The ratio of turbulent heat diffusivity to eddy diffusivity $\alpha$ is shown to be a function of $z / L$ and the formula $(2.10)$ describing qualitatively its effect is proposed. The calculated mean velocity (for unstable and stable conditions) and the mean temperature (for stable conditions assuming $\alpha=$ const.) profiles fit the experimental data $\left[{ }^{7,8}\right]$ quite satisfactorily. 